\title{
$O$ perfil do profissional fisioterapeuta atuante na unidade de terapia intensiva:
}

\section{Revisão integrativa}

\author{
The profile of the physiotherapy professional working in the unit of intensive care: Integrative \\ review
}

El perfil del profesional de fisioterapia que trabaja en la unidad de cuidados intensivos: Revisión integrativa

José Rogécio de Sousa Almeida

ORCID: https://orcid.org/0000-0003-3722-9354 Universidade do Estado do Rio Grande do Norte, Brasil E-mail: rogeciofisio@gmail.com

Lucas Schabarum

ORCID: https://orcid.org/0000-0003-1640-7991 Faculdade do Vale do Jaguaribe, Brasil E-mail: lucas.schabarum@gmail.com

Gleiciane Silva de Aguiar

ORCID: https://orcid.org/0000-0003-4491-5328 Faculdade do Vale do Jaguaribe, Brasil

E-mail: gleicianesilvadeaguiar@gmail.com

Jeffeson Hildo Medeiros de Queiroz

ORCID: https://orcid.org/0000-0002-3209-5679 Universidade Federal do Ceará, Brasil E-mail: jeffesonrcc@gmail.com

Elanny Mirelle da Costa

ORCID: https://orcid.org/0000-0002-2947-7529 Universidade do Estado do Rio Grande do Norte, Brasil E-mail: elannycosta@ alu.uern.br

Lucidio Clebeson de Oliveira

ORCID: https://orcid.org/0000-0002-2033-7546 Universidade do Estado do Rio Grande do Norte, Brasil E-mail: lucidioclebeson@uern.br

\begin{abstract}
Resumo
O estudo sobre perfil do profissional de fisioterapia que atua na unidade de terapia intensiva no Brasil é relevante para possibilitar um conhecimento amplo sobre quem são estes profissionais e assim desenvolver mecanismos que os ajudem nos campos laboral e intelectual. Objetivou-se conhecer o perfil dos fisioterapeutas atuantes na unidade de terapia intensiva no Brasil a partir de seu retrato sociodemográfico e profissional. Tratou-se de uma revisão integrativa desenvolvida no período de novembro de 2020 a fevereiro de 2021, utilizando o a estratégia de busca "Fisioterapia", "Serviço Hospitalar de Fisioterapia" e "Unidades de Terapia Intensiva", realizando a interação entre os descritores através do operador booleano AND. Identificou-se um cenário de profissionais de ambos os sexos, em sua maioria mulheres. Observou-se a necessidade de aprofundamento acadêmico após a graduação. A maior parte possui especialização em cardiorrespiratória. Viu-se que o retrato dos profissionais atuante na fisioterapia em unidade de terapia intensiva no país é satisfatório, mas precisa-se permanente especialização na área assim como melhoria nas garantias de direitos e valorização do trabalho.
\end{abstract}

Palavras-chave: Fisioterapeuta; Perfil; UTI; Cardiorrespiratória.

\begin{abstract}
The study on the profile of the physiotherapy professional who works in the intensive care unit in Brazil is relevant to enable a broad knowledge of who these professionals are and thus develop mechanisms that help them in the labor and intellectual fields. The objective was to know the profile of physical therapists working in the intensive care unit in Brazil from their sociodemographic and professional portrait. It was an integrative review developed from November 2020 to February 2021, using the search strategy "Physiotherapy", "Hospital Physical Therapy Service" and "Intensive Care Units", performing the interaction between the descriptors through the Boolean AND operator. A scenario of professionals of both sexes, mostly women, was identified. There was a need for further academic development after graduation. Most have specialization in cardiorespiratory. It was seen that the portrait of
\end{abstract}


professionals working in physiotherapy in an intensive care unit in the country is satisfactory, but permanent specialization in the area is needed, as well as improvement in the guarantees of rights and valuation of work.

Key words: Physiotherapist; Profile; ICU; Cardiorespiratory.

\section{Resumen}

El estudio sobre el perfil del profesional de fisioterapia que trabaja en la unidad de cuidados intensivos en Brasil es relevante para permitir un conocimiento amplio de quiénes son estos profesionales y así desarrollar mecanismos que los ayuden en los campos laboral e intelectual. El objetivo fue conocer el perfil de los fisioterapeutas que trabajan en la unidad de cuidados intensivos en Brasil a partir de su retrato sociodemográfico y profesional. Se trató de una revisión integradora desarrollada desde noviembre de 2020 a febrero de 2021, utilizando la estrategia de búsqueda "Fisioterapia", "Servicio de Terapia Física Hospitalaria" y "Unidades de Cuidados Intensivos", realizando la interacción entre los descriptores a través del operador booleano AND. Se identificó un escenario de profesionales de ambos sexos, en su mayoría mujeres. Era necesario un mayor desarrollo académico después de la graduación. La mayoría tiene especialización en cardiorrespiratorio. Se constató que el retrato de los profesionales que laboran en fisioterapia en una unidad de cuidados intensivos en el país es satisfactorio, pero se requiere una especialización permanente en el área, así como una mejora en las garantías de derechos y valoración del trabajo.

Palabras clave: Fisioterapeuta; Perfil; UCI; Cardiorrespiratorio.

\section{Introdução}

Recém completados 50 anos do seu reconhecimento como curso de nível superior, a fisioterapia sofreu grandes mudanças legais e científicas, em que o profissional dos dias de hoje pouco se compara ao "técnico de reabilitação" dos anos 50. Hoje atuante como profissional da saúde na promoção, prevenção e recuperação de saúde, com vasta área de atuação em diferentes áreas de especialização e níveis de apoio à saúde, que vai da atenção primária até atendimento mais complexo (Shiwa; Schimitt e João, 2016). Existem relatos de que a fisioterapia atua no cenário de Unidades de Terapia Intensiva (UTIs) desde os anos 70, na qual alguns anos após, foi efetivamente consolidado após uma portaria do Ministério da Saúde (MS), enfatizando a necessidade de um fisioterapeuta para cada dez leitos e após os anos se passando, a fisioterapia só ganhou mais destaque e espaço (Filho et al., 2021).

Segundo Santos et al. (2014) a profissão de fisioterapeuta faz parte da rede de atendimento multidisciplinar a pacientes que se encontram em uma unidade de terapia intensiva (UTI). Sua execução é ampla e se apresenta em várias divisões do tratamento intensivo. O delineamento do intensivista vai além da fisioterapia pneumofuncional e neurofuncional. Sua colaboração está associada também com processos complexos na UTI, como por exemplo ventilação mecânica, atendimento a parada cardiorrespiratória, e em processos de intubação também. Assim, é essencial um fisioterapeuta na UTI, tendo em vista que este profissional consegue melhorar o funcionamento respiratório e musculoesquelético do paciente hospitalizado, por meio de alguns recursos manuais, como a mobilização precoce. Diminuindo assim, o comprometimento funcional de paciente críticos (Paulo et al., 2021).

Segundo Ceregato, Ribeiro e Souza (2021), os profissionais atuantes na área hospitalar, principalmente em UTI, devem possuir conhecimentos essenciais de forma teórico-prático com o objetivo de conseguir lidar com as situações adversas que possam ser encontradas neste cenário, com o objetivo de minimizar complicações causadas por internações extensas. $\mathrm{O}$ papel que o fisioterapeuta desempenha na UTI é variável, mas na maioria das UTIs do Brasil, tem como finalidade manter a funcionalidade dos pacientes através da prevenção e tratamento de mudanças osteomioarticulares e de distúrbios respiratórios, além de monitorar trocas gasosas, acompanhar a monitorização da ventilação mecânica, gerenciar ventilação invasiva e não invasiva entre outros (Menezes, 2011).

A implantação do fisioterapeuta nesta área se deu início ao final dos anos 70 e desde então sua importância dentro da equipe na UTI vem crescendo. Nos anos 2000 o Conselho Federal de Fisioterapia e Terapia Ocupacional (COFFITO) admitiu os primeiros cursos de Fisioterapia Intensiva e semi intensiva no Brasil (Russo et al., 2012). 
As UTIs neonatais foram desenvolvidas com finalidade de entregar atendimento aos recém-nascidos (RNs) prematuros. O profissional de fisioterapia integra também a essa equipe multidisciplinar presente nas UTIs neonatais, tendo papel definido sobre as funções respiratórias e motoras dos neonatos. De acordo com a portaria $\mathrm{N}^{\mathrm{o}} 3.432$ do Ministério da Saúde, em vigor desde 12 de agosto de 1998, impõe que hospitais terciários devem conter fisioterapeutas dentro da UTI em período integral (Arakaki et al., 2016).

A fisioterapia voltada para a área respiratória atua na prevenção e no tratamento de doenças utilizando técnicas e procedimentos terapêuticos em 3 níveis (ambulatorial, hospitalar e na unidade de terapia intensiva), com a meta de melhorar ou aumentar a função pulmonar, através de técnicas de desobstrução brônquica, expansão pulmonar e equilíbrio da ligação ventilação/perfusão, com o intuito de diminuição do tempo de internação hospitalar (Alves, 2012).

Portanto, diante ao grande crescimento da fisioterapia, principalmente no âmbito hospitalar e de terapia intensiva, se vê de grande importância estudar o perfil dos profissionais de fisioterapia que atuam na terapia intensiva no país, assim propõe-se responder a seguinte pergunta problema: qual o perfil do profissional de fisioterapia que atua na unidade de terapia intensiva no Brasil?

Deste modo, objetiva-se pesquisar e identificar o perfil do fisioterapeuta atuante na UTI, assim como mostrar as extensões em que mais atuam apontando suas especialidades e traçar suas atribuições dentro da UTI.

\section{Metodologia}

Este estudo se caracteriza como uma revisão integrativa (Botelho, Cunha e Macedo, 2011) com o objetivo de responder a seguinte pergunta norteadora: "Qual o perfil do profissional de fisioterapia que atua na unidade de terapia intensiva no Brasil?

O processo de seleção e buscas de estudos aconteceu no período de novembro de 2020 a fevereiro 2021, e foi realizado nas seguintes bases: SCIELO, PUBMED, LILACS, WOSCIENCE. Os critérios de inclusão para busca dos estudos foram: a) que se encaixem no tema de fisioterapia na unidade de terapia intensiva, artigos em inglês e português, encontrados nas bases indicadas; b) publicados entre 2008 a 2020; c) disponíveis como textos completos e d) escritos em língua portuguesa, inglesa ou espanhola. Descartou-se os estudos que: a) não estiverem disponíveis de forma gratuita; b) não se enquadrassem como artigo; c) não tivesse relação direta com a temática pesquisada e d) não fossem realizados no Brasil.

No processo de buscas, usou-se termos do DeCS (Descritores em Ciências da Saúde) e do MeSH (Medical Subject Headings), selecionados anteriormente e adequados à pergunta científica que direcionou este estudo. Os descritores selecionados foram "Fisioterapia", "Serviço Hospitalar de Fisioterapia" e "Unidades de Terapia Intensiva", realizando a interação entre os descritores através do operador booleano AND.

Os filtros utilizados foram "ano de publicação", "texto livre", "línguas", "humanos" e "país de origem", a partir dos filtros disponíveis em cada base de dados online e de acordo com os critérios de seleção dos estudos previamente estabelecidos.

Após o processo de busca e seleção dos estudos, elegeram-se 35 para análise. Desses estudos, após leitura detalhada e aproximando-a da questão diretriz deste artigo e dos pré-requisitos para sua inclusão, estabeleceu-se um total final de nove produções (ver fluxograma - Figura 1).

A seleção dos textos completos e de suas informações foi realizada a partir da ficha URSi e do fluxograma do processo de seleção dos textos com a utilização do Checklist PRISMA. 
Figura 1. Fluxograma do processo de análise dos estudos.

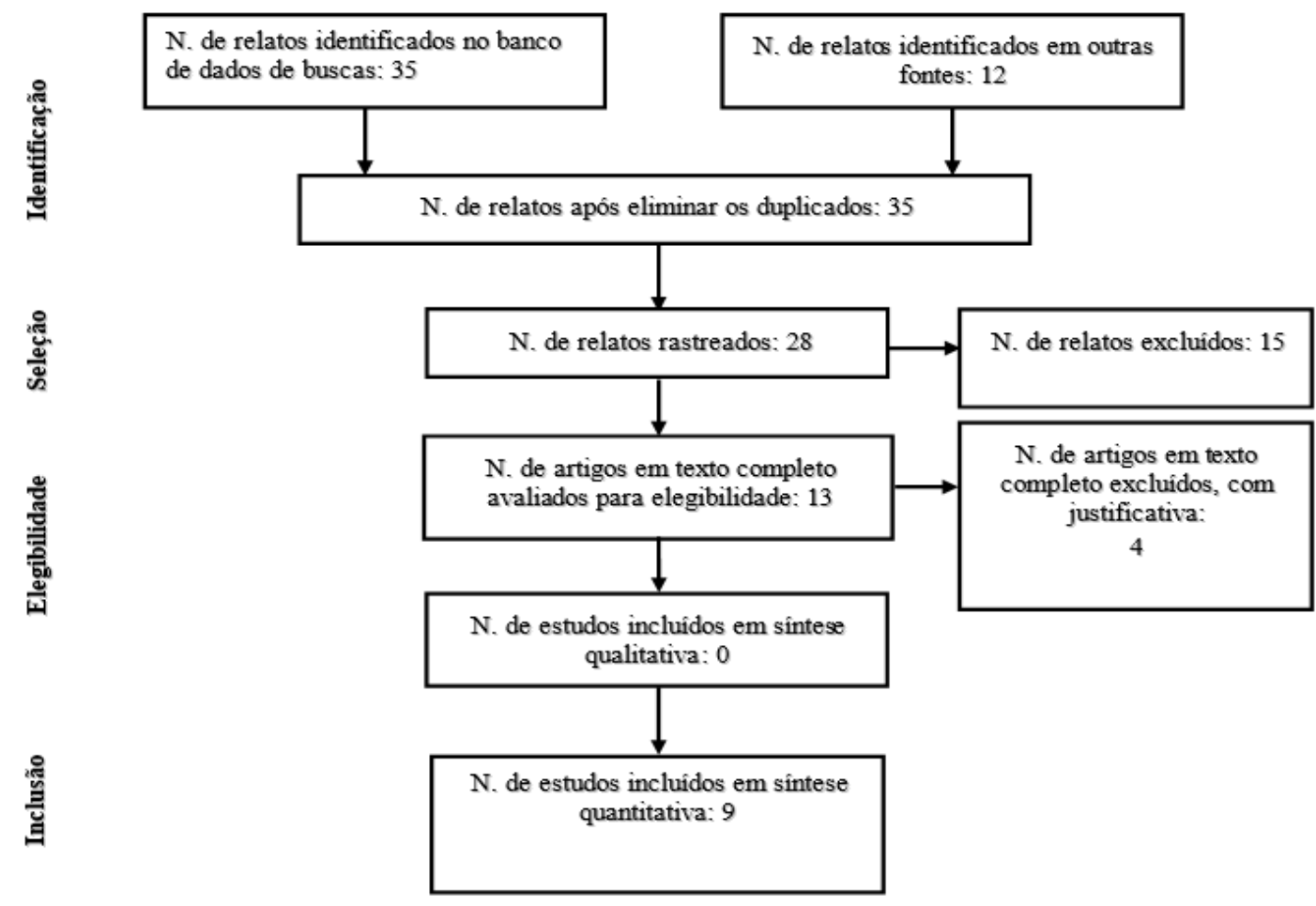

Fonte: Autores (2021).

\section{Resultados}

Os estudos selecionados foram revisados e dispostos no Quadro 1 (ver o quadro) em relação a autoria, ano de publicação, objetivo, tipo de estudo, instrumentos utilizados na coleta de dados, principais resultados e conclusões. Identificouse artigos publicados no período de 2003 a 2020 com pesquisas realizadas em Paraíba, Pernambuco e São Paulo, todos com tipo de estudo transversal, com um quantitativo de participantes variando entre 122 a 1558.

Observou-se uma variedade nos instrumentos metodológicos aplicados para atingir o objetivo do estudo, como questionário semiestruturado, questionário autoaplicável, questionário estruturado, questionário virtual e uma entrevista com perguntas fechadas e objetivas.

Percebeu-se a presença de pessoas de ambos os sexos contidas nos diversos estudos, ficando evidente o maior número de mulheres em relação os homens, especialmente em Soares et al. (2016) que chegou a 94\% dos participantes. Destaca-se ainda o interesse dos profissionais fisioterapeutas em não estagnar seus estudos logo após a graduação, buscando assim por pós-graduações e/ou especializações existentes na área de atuação, como visto em Costa et al. (2008) o qual evidenciou a opção de $71 \%$ por especialização em cardiorrespiratória.

Este abrir-se às descobertas acadêmicas após a graduação mostra um perfil mais jovem de fisioterapeutas dentro de um processo de crescimento profissional, são pessoas que compõem quadros funcionais hospitalares bem preparadas e bastante qualificadas, como afirma Liberali, Davidson e Santos (2014). Assim, nota-se que há obtenção de renda mensal melhor destes profissionais, como destacados por Shiwa, Schimitt e João, 2016 
Quadro 1. Síntese dos estudos analisados em relação a objetivo, tipo de estudo, amostra, intervenções, resultados e conclusões.

\begin{tabular}{|c|c|c|c|c|c|}
\hline $\begin{array}{l}\text { Autores, } \\
\text { ano }\end{array}$ & Objetivo & Tipo de estudo & $\begin{array}{l}\text { Intervenções/instrumentos } \\
\text { de coleta }\end{array}$ & Resultados & Conclusões \\
\hline $\begin{array}{l}\text { Alves et } \\
\text { al., } 2020 .\end{array}$ & $\begin{array}{l}\text { Analisar o perfil } \\
\text { dos } \\
\text { fisioterapeutas } \\
\text { que atuam nas } \\
\text { UTIs adulto da } \\
\text { cidade de } \\
\text { Teresina/PI. }\end{array}$ & $\begin{array}{l}\text { estudo de campo, } \\
\text { quantitativo, } \\
\text { exploratório e } \\
\text { transversal. }\end{array}$ & $\begin{array}{l}\text { questionário online com } \\
\text { questões objetivas. }\end{array}$ & $\begin{array}{l}58,5 \% \text { eram } \\
\text { mulheres; } 92,45 \% \\
\text { possuem pós- } \\
\text { graduação e destas } \\
18,9 \% \text { possuem } \\
\text { mestrado; salienta- } \\
\text { se que } 16,9 \% \text { não } \\
\text { possuem graduação } \\
\text { na área de terapia } \\
\text { intensiva. }\end{array}$ & $\begin{array}{l}\text { Possuem } \\
\text { qualificação } \\
\text { necessária. Os } \\
\text { profissionais de } \\
\text { instituições privadas } \\
\text { possuem maior } \\
\text { formação } \\
\text { intensivista e grau } \\
\text { de satisfação } \\
\text { profissional. }\end{array}$ \\
\hline $\begin{array}{l}\text { Araújo e } \\
\text { Júnior, } \\
2003 .\end{array}$ & $\begin{array}{l}\text { Traçar um perfil } \\
\text { dos profissionais } \\
\text { da fisioterapia } \\
\text { que trabalham em } \\
\text { UTI. }\end{array}$ & $\begin{array}{l}\text { pesquisa de campo, } \\
\text { transversal e } \\
\text { quantitativa }\end{array}$ & Questionários estruturado. & $\begin{array}{l}74 \% \text { do sexo } \\
\text { feminino. Se o } \\
\text { paciente tem } \\
\text { autonomia sobre } \\
\text { sua própria vida e } \\
74 \% \text { disseram que } \\
\text { não. } 94 \% \text { contra a } \\
\text { eutanásia ativa e } \\
74 \% \text { contra a } \\
\text { eutanásia passiva. }\end{array}$ & $\begin{array}{l}40 \% \text { dos } \\
\text { fisioterapeutas de } \\
\text { Alagoas exercem } \\
\text { sua atividade em } \\
\text { UTI intensiva, 100\% } \\
\text { dos participantes } \\
\text { afirmam fornecer } \\
\text { todas as informações } \\
\text { relacionadas ao } \\
\text { tratamento do } \\
\text { paciente. }\end{array}$ \\
\hline $\begin{array}{l}\text { Badaró e } \\
\text { Guilhem, } \\
2011 .\end{array}$ & $\begin{array}{l}\text { Conhecer o perfil } \\
\text { sociodemográfico } \\
\text { e profissional dos } \\
\text { fisioterapeutas } \\
\text { que atuam na } \\
\text { cidade de Santa } \\
\text { Maria, RS. }\end{array}$ & $\begin{array}{l}\text { transversal e } \\
\text { descritivo, com } \\
\text { abordagem } \\
\text { quantitativa }\end{array}$ & $\begin{array}{l}\text { questionário } \\
\text { semiestruturado }\end{array}$ & $\begin{array}{l}167 \text { fisioterapeutas } \\
\text { jovens, homens e } \\
\text { mulheres, do } \\
\text { serviço público e } \\
\text { privado. }\end{array}$ & $\begin{array}{l}\text { Predominam jovens. } \\
\text { O crescimento da } \\
\text { profissão é } \\
\text { constante. } \\
\text { Necessidade de } \\
\text { trabalhar questões } \\
\text { éticas. }\end{array}$ \\
\hline $\begin{array}{l}\text { Costa et } \\
\text { al., } 2008 .\end{array}$ & $\begin{array}{l}\text { Investigar o perfil } \\
\text { dos } \\
\text { fisioterapeutas } \\
\text { que atuam nas } \\
\text { unidades de } \\
\text { terapia intensiva } \\
\text { (UTIs) no Brasil. }\end{array}$ & $\begin{array}{l}\text { pesquisa de campo, } \\
\text { prospectiva, } \\
\text { transversal, com } \\
\text { abordagem } \\
\text { predominantemente } \\
\text { quantitativa. }\end{array}$ & $\begin{array}{l}\text { questionário } \\
\text { semiestruturado }\end{array}$ & $\begin{array}{l}\text { Maioria gestores } \\
\text { alocados nas UTIs } \\
\text { e com } \\
\text { especialização em } \\
\text { cardiorrespiratória. }\end{array}$ & $\begin{array}{l}\text { Caracteriza-se por } \\
\text { profissionais } \\
\text { qualificados. }\end{array}$ \\
\hline $\begin{array}{l}\text { Gomes et } \\
\text { al., } 2010 \text {. }\end{array}$ & $\begin{array}{l}\text { Analisar o perfil } \\
\text { dos } \\
\text { fisioterapeutas } \\
\text { atuantes em } \\
\text { unidades de } \\
\text { terapia intensiva } \\
\text { da cidade de } \\
\text { Maceió/AL. }\end{array}$ & $\begin{array}{l}\text { pesquisa de campo, } \\
\text { transversal e } \\
\text { descritiva }\end{array}$ & questionário autoaplicável & $\begin{array}{l}\text { Maioria mulheres e } \\
\text { com especialização, } \\
\text { ganhando entre } 5 \text { e } \\
6 \text { salários mínimos. }\end{array}$ & $\begin{array}{l}\text { Constitui } \\
\text { predominantemente } \\
\text { de adultos jovens, } \\
\text { mulheres e com } \\
\text { especialização em } \\
\text { Cardiorrespiratória. }\end{array}$ \\
\hline $\begin{array}{l}\text { Liberali, } \\
\text { Davidson } \\
\text { e Santos, } \\
2014 .\end{array}$ & $\begin{array}{l}\text { Delinear o perfil } \\
\text { dos } \\
\text { fisioterapeutas } \\
\text { que trabalham em } \\
\text { unidades de } \\
\text { terapia intensiva } \\
\text { na cidade de São } \\
\text { Paulo. }\end{array}$ & Transversal & $\begin{array}{l}\text { entrevistas com perguntas } \\
\text { fechadas e objetivas. }\end{array}$ & $\begin{array}{l}\text { Não houve } \\
\text { diferenças de } \\
\text { formação } \\
\text { acadêmica, } \\
\text { treinamento } \\
\text { profissional, } \\
\text { desempenho e } \\
\text { tempo de } \\
\text { treinamento entre } \\
\text { privado e público. }\end{array}$ & $\begin{array}{l}\text { Observou-se que os } \\
\text { fisioterapeutas } \\
\text { possuíam, em sua } \\
\text { maioria, } \\
\text { especialização na } \\
\text { área e realizam } \\
\text { regularmente cursos } \\
\text { de atualização. }\end{array}$ \\
\hline
\end{tabular}


Continuação Quadro 1.

\begin{tabular}{|c|c|c|c|c|c|}
\hline $\begin{array}{l}\text { Autores, } \\
\text { ano }\end{array}$ & Objetivo & Tipo de estudo & $\begin{array}{l}\text { Intervenções/instrumentos } \\
\text { de coleta }\end{array}$ & Resultados & Conclusões \\
\hline $\begin{array}{l}\text { Russo R.C. } \\
\text { et al., } 2012 .\end{array}$ & $\begin{array}{l}\text { Delinear o } \\
\text { perfil de } \\
\text { formação } \\
\text { profissional dos } \\
\text { fisioterapeutas } \\
\text { que atuam nas } \\
\text { unidades de } \\
\text { terapia intensiva } \\
\text { da cidade de } \\
\text { Maceió. }\end{array}$ & Transversal & $\begin{array}{l}\text { questionário auto-aplicável } \\
\text { com questões de múltipla } \\
\text { escolha. }\end{array}$ & $\begin{array}{l}54 \text { fisioterapeutas } \\
\text { jovens, maioria } \\
\text { mulheres, } 94,44 \% \\
\text { pós graduados } \\
\text { sendo } 66,67 \% \\
\text { especialistas. }\end{array}$ & $\begin{array}{l}\text { Apresentam um } \\
\text { perfil compatível } \\
\text { com a atuação em } \\
\text { unidades de terapia } \\
\text { intensiva. Em sua } \\
\text { maioria possuem } \\
\text { titulação adequada, } \\
\text { preferencialmente } \\
\text { na área de } \\
\text { Cardiorrespiratória/ } \\
\text { Terapia Intensiva. }\end{array}$ \\
\hline $\begin{array}{l}\text { Shiwa; } \\
\text { Schimitt e } \\
\text { João, } 2016\end{array}$ & $\begin{array}{l}\text { Descrever o } \\
\text { perfil do } \\
\text { fisioterapeuta } \\
\text { do Estado de } \\
\text { São Paulo. }\end{array}$ & Observacional & $\begin{array}{l}\text { questionário virtual } \\
\text { composto questões de } \\
\text { múltipla escolha e abertas. }\end{array}$ & $\begin{array}{l}80 \% \text { eram do sexo } \\
\text { feminino, } 87 \% \\
\text { optaram por } \\
\text { realizar algum } \\
\text { curso após a } \\
\text { graduação. Apenas } \\
17,9 \% \text { na área de } \\
\text { cardiorrespiratória. }\end{array}$ & $\begin{array}{l}\text { há predominância } \\
\text { feminina; jovens, } \\
\text { com pós-graduação } \\
\text { lato sensu; o tempo } \\
\text { de formação e } \\
\text { experiência são } \\
\text { relevantes para } \\
\text { melhor renda } \\
\text { mensal. }\end{array}$ \\
\hline $\begin{array}{l}\text { Soares et al., } \\
2016 .\end{array}$ & $\begin{array}{l}\text { Analisar o perfil } \\
\text { dos } \\
\text { fisioterapeutas } \\
\text { atuantes em na } \\
\text { UTI do hospital } \\
\text { regional do } \\
\text { baixo amazonas } \\
\text { (HRBA) do } \\
\text { oeste do Pará, } \\
\text { Brasil. }\end{array}$ & $\begin{array}{l}\text { pesquisa } \\
\text { quantitativa, } \\
\text { descritiva, } \\
\text { transversal. }\end{array}$ & Questionário autoaplicável & $\begin{array}{l}86,7 \% \text { do sexo } \\
\text { feminino. } 93,3 \% \\
\text { dos pesquisados } \\
\text { possuem pós- } \\
\text { graduação e dessas } \\
\text { 33\% em UTI. }\end{array}$ & $\begin{array}{l}\text { Os fisioterapeutas } \\
\text { intensivistas } \\
\text { possuem titulação } \\
\text { adequada, } \\
\text { preferencialmente } \\
\text { na área de } \\
\text { Cardiorrespiratória, } \\
\text { Terapia Intensiva. }\end{array}$ \\
\hline
\end{tabular}

Fonte: Autores (2021).

\section{Discussão}

De acordo com o estudo realizado por Badaró e Guilhem (2011), a faixa etária dos profissionais variou de 22 a 58 anos, apresentando o maior número deles até 30 anos, seguido de 31 a 40 anos. Já referente ao sexo a maioria se apresentou do sexo feminino, com uma proporção de 5 profissionais mulheres para 1 profissional do sexo masculino. Já no estudo de Silva et al. (2018), referente a idade a maioria apresentou uma média de 35,9 e também apresentando o maior número de profissionais mulheres com certa de aproximadamente $74 \%$. Este estudo mostrou resultados semelhantes quando se fala em gênero o sexo feminino apresenta maior número de profissionais dentro das UTI's e quanto a faixa etária mostra uma pequena semelhança entre 30 anos acima.

De acordo com o estudo feito por Gomes et al. (2010) o tempo de graduação dos profissionais entrevistados ficam entre 6,4 $\pm 5,7$ anos e quanto ao grau a acadêmico um pouco mais de $80 \%$ apresentam alguma especialização sendo seguido de apenas graduação e por menor número apresentando mestrado, sendo a especialidade em maior número entre eles a de cardiorrespiratória seguida de terapia intensiva.

Segundo o estudo realizado por Silva et al. (2018) o maior número de profissionais que se graduaram e atuam na área é de mais de 10 anos atuantes, também contando com o maior número entre os que possuem especialização na área. Comparando com este estudo, nota-se que a maioria dos profissionais tem pelo menos 5 anos de graduação, e em sua grande maioria de profissionais possuindo algum tipo de especialização em que atuam na UTI. 
No trabalho realizado por Alves et al. (2020), cerca de $75 \%$ dos profissionais tem uma carga semanal de trabalho de 30 horas, na sua grande maioria em setor público, logo seguidos de profissionais que tem 20 horas semanais em sua carga semanal. Já no trabalho realizado por Santos et al. (2014) também se constatou que o número de profissionais com carga de 30 horas semanais tem em torno de $75 \%$ seguidos logo de profissionais que atuam cerca de 40 horas semanais. Com base nesse estudo mostra a semelhança da carga semanal de trabalho dos profissionais atuantes na UTI, sendo a grande maioria atuando 30 horas semanais, e os outros em números menos expressivos atuando cargas semanais diferentes.

Soares et al. (2016) observou que entre os profissionais entrevistados na sua pesquisa mais de $90 \%$ fizeram uma pós-graduação, sendo a mais escolhida em UTI, logo seguida de especialização na área hospitalar e cardiorrespiratória consequentemente. Já segundo o estudo realizado por Russo et al. (2012), dentre os profissionais entrevistados a especialização mais feita por eles com cerca de $70 \%$ foi a de terapia intensiva e cardiorrespiratória, seguidamente de gerontologia e outros.

Corroborando com o presente estudo Braz, Martins e Junior (2009) afirmam que dentre os profissionais entrevistados apenas cerca de $28 \%$ não possuem nenhuma especialização, e certa de $71 \%$ possuem alguma especialização na área respiratória e/ou hospitalar. Mostrando juntamente com os outros autores que a maioria dos profissionais atuantes na UTI possuem alguma especialização na área.

Em relação a remuneração, Badaró e Guilhem (2011) evidenciou que em sua maioria com quase $45 \%$ dos profissionais são remunerados em até 4 salários mínimos, quando cerda de 38\% referem renda de 9 salários mínimos acima. Já segundo sua pesquisa, Gomes et al. (2010), a maioria dos profissionais questionados apresentam renda de 5 a 6 salários mínimos em âmbito intensivo, já na sua grande minoria apresentam uma renda superior a 9 salários, assim diferenciando da pesquisa citada anteriormente. Assim mostrando que quanto a remuneração vai variar de acordo com região e também vínculo trabalhista, mas tendo uma média de 5 salários mínimos para mais.

Segundo o estudo realizado por Shiwa, Schimitt e João (2016) apenas aproximadamente 15\% dos profissionais participantes do estudo apresentavam algum tipo de vínculo trabalhista como associação ou sindicato, e mais de $80 \%$ não apresentavam nenhum tipo de vínculo. Já no estudo de Santos et al. (2014) com cerca de 40\% dos fisioterapeutas questionados apresentam vínculo empregatício do tipo CLT, seguido de profissionais que são terceirizados, e o menor número apresentado por profissionais com vínculos em cooperativas. Mostrando nesse presente estudo que o a forma de vínculo de profissionais que atuam na UTI varia muito de região e hospital, podendo até não ter vínculo algum.

\section{Considerações Finais}

Pode-se concluir que o perfil dos fisioterapeutas atuantes no cenário nacional quanto a terapia intensiva caracterizase por profissionais qualificados, na sua grande parte com algum tipo de especialização na área, ou algum curso profissionalizante. Observou-se também que a maioria os profissionais são do sexo feminino e com mais de 5 anos de experiência.

Notou-se que a maioria dos fisioterapeutas são remunerados com valores bem variantes, não tendo um padrão muito conciso, variando de 4 e até mais de 10 salários mínimos, tendo essa variação de local e carga horária de cada um. Em relação a vínculos de trabalho, a maior parte dos profissionais não tem nenhum tipo de vínculo com algum sindicato ou associação.

Assim, conclui-se que quanto a qualificação e em relação a gênero mostra que constitui num padrão para todo o Brasil, mas quando se fala em remuneração já apresenta uma disparidade, estando relacionado a local e estado o qual se trabalha. Destaca-se ainda que muitos profissionais não possuem nenhum tipo de vínculo trabalhista formal, ressaltando haver uma defasagem e desvalorização quanto ao seguro do trabalhador fisioterapeuta intensivista e hospitalar.

Por fim, é necessário reforçar a necessidade constante de estudos que avaliem o perfil e a atuação dos profissionais fisioterapeutas nas mais diversas áreas a fim de se conhecer o modo em que estão vivenciando sua profissão e assim poder-se 
refletir sobre suas necessidades e buscar estratégias de valorização e efetivação profissional. Dessa forma, sugere-se mais pesquisas quem envolvam o levantamento do perfil do profissional fisioterapeuta que atua no cenário intensivista brasileiro e as questões relacionadas a sua atuação e condições de trabalho.

\section{Referências}

Alves, A. N. (2012). A importância da atuação do fisioterapeuta no ambiente hospitalar. Ensaios e Ciência: Ciências Biológicas, Agrárias e da Saúde, Campo Grande. 16(6), 173-184.

Alves, F. A. D et al. (2020). Perfil dos fisioterapeutas nas unidades de terapia intensiva adulto. Revista Eletrônica Acervo Saúde, Teresina. 1(55).

Arakaki, V. S. N. M et al. (2017). Mapeamento demográfico e caracterização do perfil de assistência fisioterapêutica oferecida nas unidades de terapia intensiva neonatais do Rio de Janeiro. Fisioterapia Pesquisa, Rio de Janeiro. 24(2), 143-148.

Araújo, L. Z. S \& Júnior, W. A. N (2003). A bioética e a fisioterapia nas Unidades de Terapia Intensiva. Revista Fisioterapia Universidade de São Paulo, São Paulo. 10(02), 52-60.

Badaró, A. F. V. \& Guilherm, D. (2011). Perfil sociodemográfico e profissional de fisioterapeutas e origem das suas concepções de ética. Fisioterapia Movimento, Curitiba. 24(3)

Botelho, L. L. R., Cunha, C. C. A., \& Macedo, M. (2011). O método da revisão integrativa nos estudos organizacionais. Gestão e Sociedade. Belo Horizonte. $5(2), 121-136$.

Braz, P. R. P; Martins, J. O. S. O. L; Júnior, G. V. (2009). Atuação do fisioterapeuta nas unidades de terapia intensiva da cidade de Anápolis. Anuário da Produção Acadêmica Docente, Anápolis. 3(4), 119-129.

Ceregato, A. C. L.; Ribeiro, E. R.; Souza, J. M (2021). Perfil de competências de fisioterapeutas atuantes em unidades de terapia intensiva no Estado do Paraná. Revista Sustinere, Rio de Janeiro. 9(supl.1), 208-227.

Costa, D. et al. (2008). Perfil de fisioterapeutas brasileiros que atuam em unidades de terapia intensiva. Fisioterapia e Pesquisa. São Paulo. 15(2).

Filho, L. E. C. S. et al. (2021). Impacto de um vídeo educativo de sistematização para exame físico na prática discente de fisioterapia em Unidade de Terapia Intensiva. Research, Society and Development.10(5).

Gomes, C. A. F. P et al. (2010). Análise do perfil dos fisioterapeutas atuantes em unidades de terapia intensiva da cidade de Maceió/AL. Fisioterapia Brasil, Maceió. 11(3).

Liberali, J; Davidson, J; Santos, A. M. N (2014). Disponibilidade de assistência fisioterapêutica em unidades de terapia intensiva neonatal na cidade de São Paulo. Revista Brasileira de Terapia Intensiva, São Paulo. 26(1), 57-64.

Menezes, S (2011). Fisioterapia em Terapia Intensiva: uma nova denominação para uma antiga especialidade. Assobrafir Ciência. 2 (2), $49-53$.

Mohwe, D. et al. (2009). Preferred reporting items for systematic reviews and meta-analyses: The PRISMA statement. PLoS Med. 6(7).

Paulo, F. V. S et al. (2021). Mobilização precoce a prática do fisioterapeuta intensivista: intervenções e barreiras. Rev. Pesqui. Fisioter., Salvador. 11(2), 298306.

Russo, R. C. et al. (2012). Perfil de formação profissional dos fisioterapeutas das unidades de terapia intensiva da cidade de Maceió. Assobrafir Ciência, Maceió. 3(2), 21-30.

Santos, L. F. O. et al. (2014). Perfil dos fisioterapeutas que atuam em unidades de terapia intensiva na cidade de Salvador/Bahia. 2014. 21p. Trabalho de conclusão da pós-graduando em Fisioterapia Hospitalar-EBMSP.

Shiwa, S. R.; Schimitt, A. C. B.; João, S. M. A. (2010). O fisioterapeuta do estado de São Paulo. Fisioterapia Pesquisa, São Paulo. 23(3).

Silva, R. A. D. et al. (2018). Síndrome de Burnout: realidade dos fisioterapeutas intensivistas?. Fisioterapia Pesquisa, Recife. 25(4), 388-394.

Soares, M. G. D. et al. (2016). Perfil dos fisioterapeutas atuantes em 2013, na unidade de terapia intensiva do Hospital Regional do Baixo Amazonas do oeste do Pará, Brasil. EFD esportes.com: Revista Digital, Buenos Aires. 20(212).

Ursi, E. S. (2005). Prevenção de lesões de pele no perioperatório: revisão integrativa da literatura. 2005.130 f. Dissertação (Mestrado em Enfermagem) Escola de Enfermagem, Universidade de São Paulo, Ribeirão Preto. 